\title{
A Stochastic Framework to Model Bending of Textile Antennas
}

\author{
(Invited Paper)
}

\author{
Hendrik Rogier \\ INTEC-IMEC \\ Ghent University \\ Ghent, Belgium \\ Hendrik.Rogier@intec.ugent.be
}

\begin{abstract}
The polynomial chaos expansion is combined with a dedicated cylindrical cavity model to quantify the statistical variations in textile antenna performance under random bending conditions.
\end{abstract}

\section{INTRODUCTION}

Wearable antennas deployed in rescue worker garments are subjected to different adverse conditions that affect their radiation performance. Currently, these effects are accounted for in the antenna design phase by overspecification, which may lead to out-of-band interference and increased cost. Hence, accurate tools are needed to quantify the statistics of the textile antenna's figures of merit due to random variations in antenna geometry. Therefore, we present a stochastic framework [1] combining the non-intrusive polynomial chaos expansion [2], [3] with an analytic model [4] for curved textile antennas to quantify variations in antenna resonance frequency when deploying the wearable antenna on persons with different morphology. An example demonstrates that CPU-time is reduced by a factor 250 compared to a Monte Carlo approach.

\section{StOchastic MODEL FOR CURVED TEXTILE ANTENNAS}

\section{A. Cavity model for bent textile antennas}

Consider a rectangular patch of length $L$, width $W$ on a textile substrate of thickness $h$ and permittivity $\epsilon_{r}$. As shown in Fig. 1, the textile antenna is subjected to cylindrical bending in the direction of the patch width with angle of curvature $R$. The resonance frequency satisfies [4]

$$
J_{k_{\phi}}^{\prime}\left(k_{\rho} R\right) Y_{k_{\phi}}^{\prime}\left(k_{\rho}(R+h)\right)=J_{k_{\phi}}^{\prime}\left(k_{\rho}(R+h)\right) Y_{k_{\phi}}^{\prime}\left(k_{\rho} R\right),
$$

with $k_{\phi}=\frac{m \pi}{\beta}, k_{z}=\frac{n \pi}{L}, k_{\rho}=\sqrt{k^{2}-k_{z}^{2}}$. Two specific effects are important when applying the model to textile antennas. First, in textile antennas the effect of bending is different when the antenna patch is implemented in a stretchable fabric, such as Tafetta, compared to a nonstretchable patch, made of copper foil. The angle $\beta$ accounts for this effect by the relationship $W=\beta(R+h d)$ with $d=0.5$ for perfectly stretchable patches and $d=1$ for completely non-stretchable patches. Second, during bending, the textile fabric implementing the antenna substrate may be compressed, altering the permittivity compared to planar state. The permittivity of the compressed substrate is given by $\epsilon_{r, \text { comp }}=\epsilon_{r, \text { flat }}\left(1+\eta \frac{h[m m](d-0.5)}{R[m]}\right)$, with $\eta$ a substratedependent empirical parameter, measured on actual bent textile antenna prototypes. Therefore for non-magnetic substrates, the resonance frequency $f_{r}$ of the bent patch is derived from the wave number $k=2 \pi f_{r} \sqrt{\mu_{0} \epsilon_{0} \epsilon_{r, \text { comp }}}$.

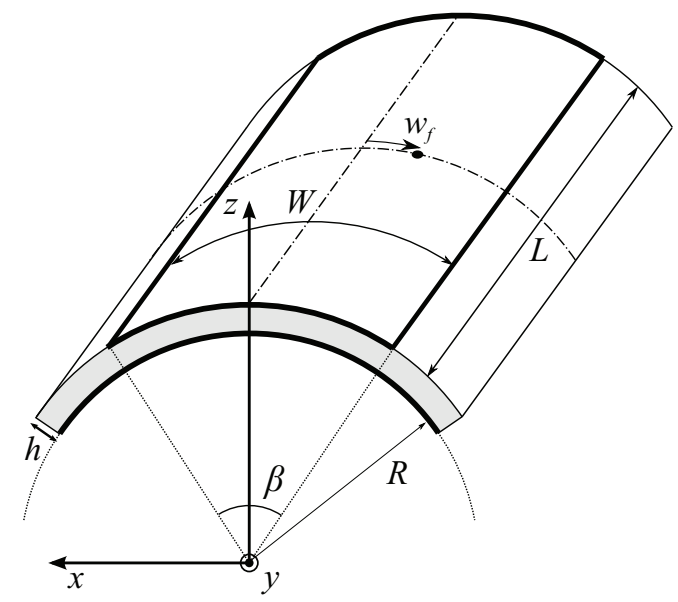

Fig. 1. Cylindrically bent textile antenna.

\section{B. Nonintrusive polynomial choas expansion}

In reality, the bending the wearable antenna undergoes when deployed on a human arm of leg will differ from person to person. Therefore, a statistical analysis is in order. The radius of curvature $R$ is then considered a random input variable and the corresponding resonance frequency $f_{r}$ will vary according to a statistical distribution. Assume that the statistical variation of $R$ is described by the cumulative distribution function $\mathcal{P}^{R}$ and its probability density function by $d \mathcal{P}^{R}$ within a certain range $\Omega$ of radii of curvature. We then apply on the WienerAskey scheme [3], [2] to approximate the transformation $f_{r}=$ $\mathbb{F}(R)$ by the following polynomial expansion $\mathbb{P}(R)$ of order $P$ [1]

$$
f_{r} \approx \mathbb{P}(R)=\sum_{k=0}^{P} f_{r, k} \phi_{k}(R) .
$$


This polynomial converges exponentially to the function $f_{r}=$ $\mathbb{F}(R)$ for $P \rightarrow \infty$ when the set of expansion polynomials forms a complete orthogonal basis in $\Omega$ with orthogonality relation

$$
\left\langle\phi_{i}(R), \phi_{j}(R)\right\rangle=\int_{\Omega} \phi_{i}(R) \phi_{j}(R) d \mathcal{P}^{R}(R)=\left\langle\left(\phi_{i}\right)^{2}\right\rangle \delta_{i j} .
$$

The Wiener-Askey scheme directly provides a number of families of orthogonal polynomials corresponding to wellestablished distributions $d \mathcal{P}^{R}(R)$, such as Hermite polynomials for the Gaussian distribution and Legendre polynomials for the uniform distribution. To determine the unknown expansion coefficients $f_{r, k}$, we apply Galerkin weighting to (2) and make use of (3) to obtain

$$
f_{r, j}=E\left[\mathbb{F}(R) \phi_{j}(R)\right]=\int_{\Omega} \mathbb{F}(R) \phi_{j}(R) d \mathcal{P}^{R}(R) .
$$

This integral is numerically evaluated by a suitable $N$-point Gauss quadrature rule, being

$$
f_{r, j} \approx \sum_{i=1}^{N} w_{i} \mathbb{F}\left(R_{i}\right) \phi_{j}\left(R_{i}\right), \quad j=0,1, \ldots, P ;
$$

with $R_{i}$ the quadrature points given by the $N$ zeros of $\phi_{N}(R)$ in $\Omega$ and $w_{i}$ the corresponding weights. In order to evaluate (5), we must calculate $f_{r}=\mathbb{F}(R)$ for $N$ realizations of the bending radius $R$ corresponding to the quadrature points.

\section{EXAMPLE}

Consider a probe-fed microstrip patch antenna of length $L=5.25 \mathrm{~cm}$ and width $W=4.37 \mathrm{~cm}$, with the probe located on the perpendicular bisector of the $L$ edge at a distance $w_{f}=1.1 \mathrm{~cm}$ from the patch center, as shown in Fig. 1 . The antenna, resonating in the vicinity of the $2.45 \mathrm{GHz}$ ISM band, is implemented on a cotton substrate (permittivity $\epsilon_{r}=1.715$, thickness $h=2.7 \mathrm{~mm}$ ) with non-stretchable copper foil $(d=1)$ serving as patch material. The resonance frequency of the prototype is first measured for different radii of curvature to determine $\eta$ in the dispersion relation, according to the procedure outlined in [4]. Then, we apply the statistical framework to the case where the textile antenna is bent around a human leg, with an average radius of curvature $\bar{R}=10 \mathrm{~cm}$. We assume that, due to variations in body morphology, the radius of curvature behaves as a uniformly distributed random variable in the interval $[2 \mathrm{~cm}, 18 \mathrm{~cm}]$. We therefore apply the non-intrusive polynomial chaos expansion (2) using an orthogonal set of Legendre polynomials, where we evaluate (5) in 25 quadrature points, requiring $2.3 \mathrm{~s}$ on an Intel ${ }^{\circledR}$ Core $^{\mathrm{TM}}$ i7-2760QM CPU operating at $2.40 \mathrm{GHz}$ with 8 GB RAM using Mathematica 9.0. Fig. 2 shows the resulting polynomial expansion for orders $P=5,10,15$, together with the quadrature points corresponding to the zeros of the Legendre polynomial of order 25 . The expansion has clearly converged for a polynomial order $P=15$. To validate our new approach we apply the Monte Carlo technique, evaluating (1) for 50000 realizations, which takes $10 \mathrm{~min} 51 \mathrm{~s}$. Fig. 3 shows a good agreement for the the probability density function obtained by both approaches.

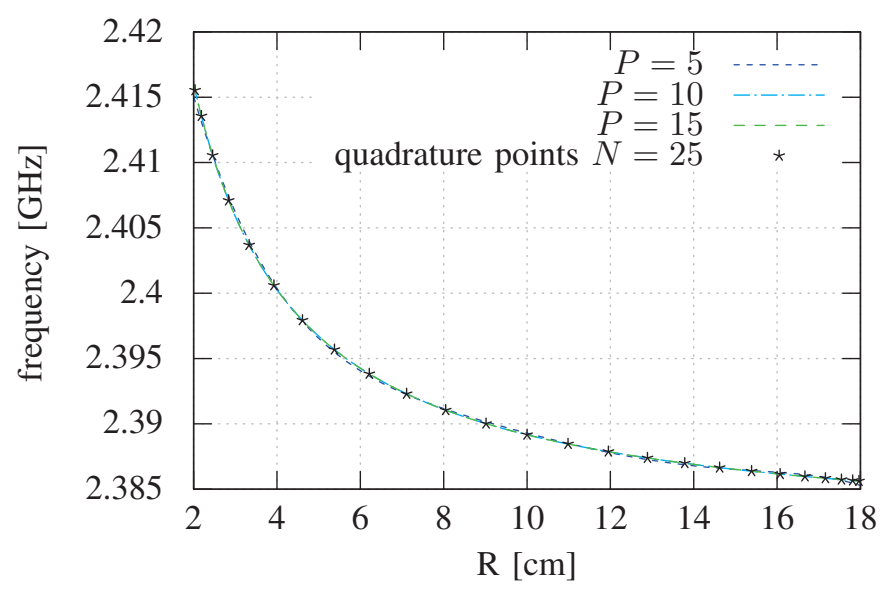

Fig. 2. Polynomial chaos expansion $\mathbb{P}(R)$ for different maximum polynomial orders $P$ for a textile antenna bent around a leg with uniformly distributed curvature radius $R$ in the range $[2,18] \mathrm{cm}$.

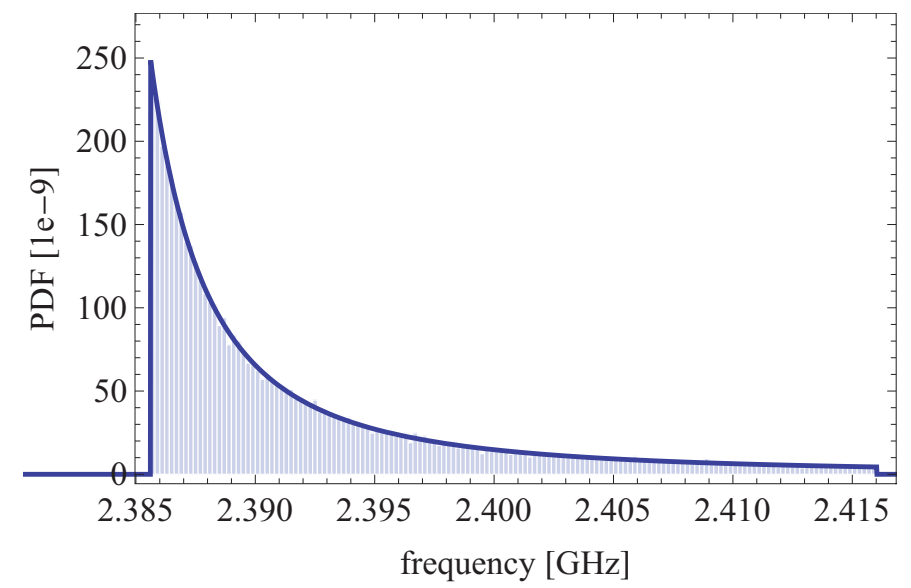

Fig. 3. Probability density function: polynomial chaos expansion (full line) versus Monte Carlo simulation (bars).

\section{ACKNOWLEDGEMENT}

Part of this work was supported by BELSPO through the IAP Phase VII BESTCOM project.

\section{REFERENCES}

[1] F. Boeykens, H. Rogier, and L. Vallozzi, "An efficient technique based on polynomial chaos to model the uncertainty in the resonance frequency of textile antennas due to bending," IEEE Trans. on Antennas and Propagation, vol. 62, no. 3, p. in press, Mar. 2014.

[2] X. Wan and G. E. Karniadakis, "Beyond Wiener-Askey expansions: Handling arbitrary PDFs," J. Sci. Comput, vol. 27, no. 1-3, pp. 455-464, 2006.

[3] D. Xiu, "Fast numerical methods for stochastic computations: A review," Commun. Comput. Phys, vol. 5, no. 2-4, pp. 242-272, February 2009.

[4] F. Boeykens, L. Vallozzi, and H. Rogier, "Cylindrical bending of deformable textile rectangular patch antennas," International Journal of Antennas and Propagation, vol. 420, no. 11, pp. 1-11, Article ID 170, doi:10.1155/2012/170 420, 2012. 